\title{
Non Hodgkin Lymphoma Presenting as a Pancreatic Mass- Treated Successfully with R-Chop
}

Krishnamani Kalpathi*

Department of Medical Oncology, Care Institute of Oncology, Hyderabad, India

Corresponding Author: Krishnamani Kalpathi, Department of Medical Oncology, Care Institute of Oncology, Hyderabad, India.

Received date: April 24, 2021; Accepted date: July 01, 2021; Published date: July 05, 2021

Citation: K Krishnamani. (2021) Non Hodgkin Lymphoma Presenting As a Pancreatic Mass- Treated Sucessfully with R-Chop. International Journal of Clinical Case Reports and Reviews. 7(4); DOI: 10.31579/2690-4861/132

Copyright: (c) 2021 Krishnamani Kalpathi, This is an open-access article distributed under the terms of the Creative Commons Attribution License, which permits unrestricted use, distribution, and reproduction in any medium, provided the original author and source are credited.

Abstract
Primary pancreatic lymphomas are extremely rare, when present mimics symptoms of adenocarcinoma of
the pancreatic head. [1] They account for less than 1\% of pancreatic neoplasms [2]. The most common
histological subtype is diffuse large B cell lymphoma (DLBCL) [3]. Herein, we present a case of a 47 year
old female who presented with symptoms of pain abdomen and weight loss. CT (Computerized Tomography)
was suggestive of a lesion in the pancreatic head which on histopathology was diagnosed as a primary non-
Hodgkin's lymphoma confirmed by immunohistochemistry.
Keywords: non hodgkins lymphoma; pancreas; dlbl

\section{Introduction}

The gastrointestinal region is the commonest site of extra nodal NHL followed by skin. Gastrointestinal non-Hodgkin lymphoma commonly involves the stomach and the small bowel. It rarely presents as a pancreatic mass accounting for less than $1 \%[1,2]$. It tends to have a male predominance (male-to-female ratio of 7:1) with age ranging from 35 to 75 years (mean age: 55 years) [4]. Primary Pancreatic Lymphomas (PPLs) are potentially treatable [5]. Majority of patients can be managed with chemotherapy and the prognosis is much better compared to patients with pancreatic adenocarcinoma which has a dismal outcome [5].

\section{Case report}

A 47 year old lady without any co morbidities presented with pain abdomen, dyspepsia and 8 kilogram weight loss of 3 months duration. She did not report any symptoms of fever, night sweats, pruritis, chest symptoms, and bowel and bladder disturbances. Clinical examination was unremarkable but for mild pallor. There was no lymphadenopathy and organomegaly. She was hemodynamically stable. Initial imaging done elsewhere with Computerised Tomography (CT) of abdomen was suggestive of a $34 \times 57 \times 66 \mathrm{~mm}$ pancreatic head lesion, with loss of fat planes with stomach. Also noted was lymphadenopathy involving peripancreatic, periportal and portocaval regions. CA 19.9 was normal (0.760 units/ml). Complete blood picture, renal, liver function tests and
LDH was normal. Viral markers were negative. An endoscopic ultrasound (EUS) was suggestive of gastric antral ulcer and a pancreatic body and tail mass. EUS guided fine needle aspiration cytology (FNAC) was done which suggested a poorly differentiated neoplasm. Immunohistochemistry (IHC) was done to further characterize the lesion which showed positivity for LCA, CD 20, BCL2, BCL6, CD 45 and CD 10 with KI-67 of 50\%. The lesion was negative for Pan CK, Chromogranin and CA 19.9 leading to a diagnosis of B cell NHL (nonHodgkin's lymphoma). Fluorescence in situ Hybridisation (FISH) done for BCL-2, BCL-6 and c-myc expression and rearrangement were negative ruling out a possibility of double hit lymphoma.

PET CT (Positron Emission Tomography Computerised Tomography) scan was done for baseline staging. PET CT was suggestive of a metabolically active locally advanced primary pancreatic head lesion infiltrating the pyloric antrum with adjacent peripancreatic nodes. Also seen were metabolically active Index score was 2 which is low intermediate. A diagnosis of Stage III BE Non-Hodgkin's lymphoma was made. Considering the prognosis and available options for treatment, she was offered R- CHOP (Rituximab-Cyclophosphamide, Doxorubicin, Vincristine and prednisolone) based chemotherapy. After three cycles, an interim PET-CT done suggested a complete metabolic response. She received a total of six cycles and continues to be in remission. 

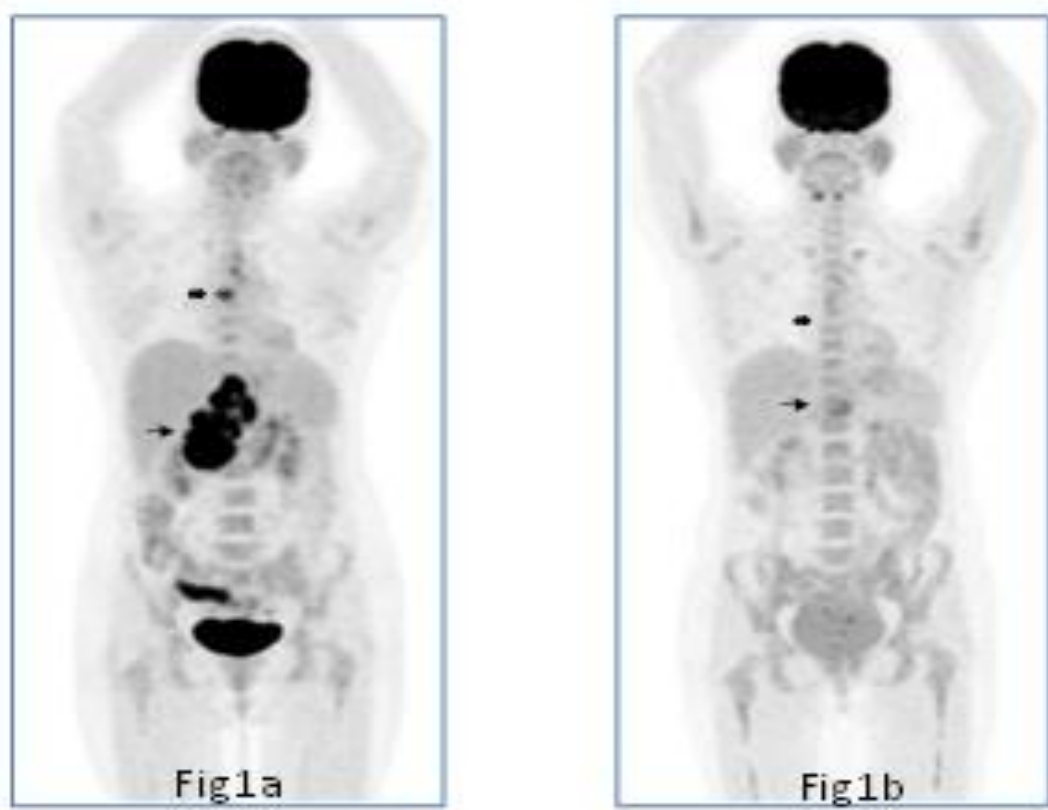

Fis la - FDG PET CTIIIP image, staging scan, shows meta bolically active b rge exophytic pancreatic mass (bbch a rrow) and metabolically acties subcarinal lymphadenopathy Eo lid a row).

Fis $1 \mathrm{~b}$ - FDG PET CT MIP image, post chemothera $p$ y scan, shows nea r complete regresion of pa ncreatic masz (bb charrow) a od a ubcarina I l/mph node (solid a rrow).
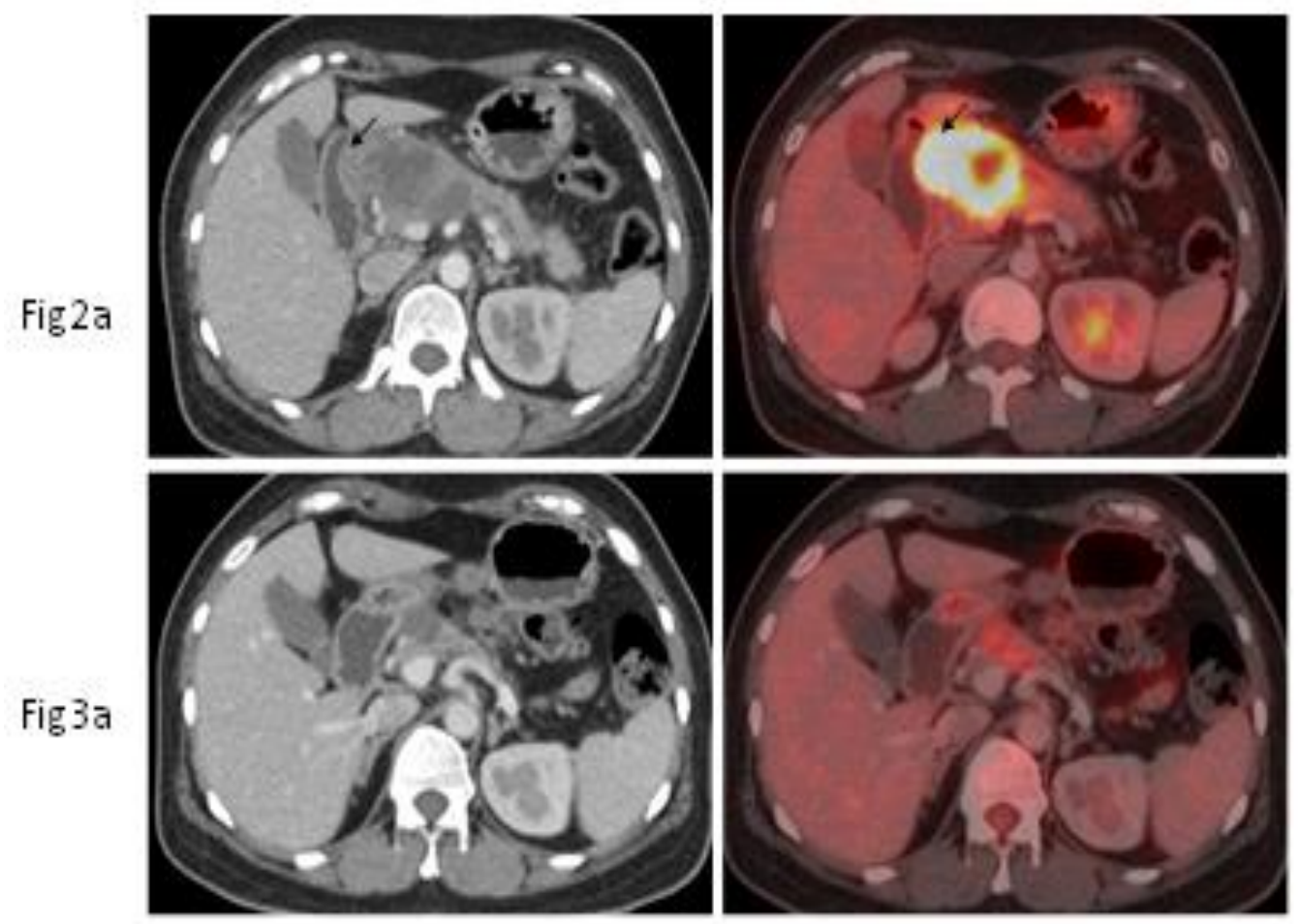

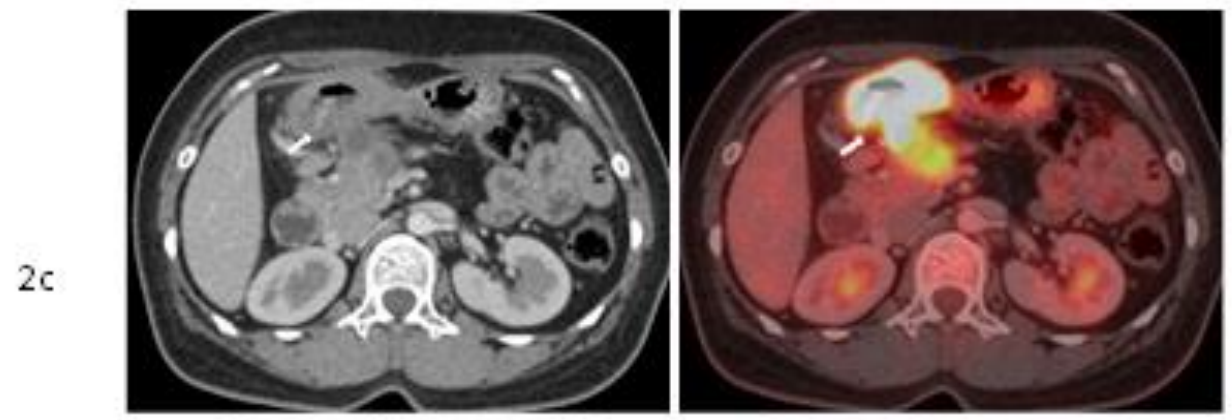

$2 d$
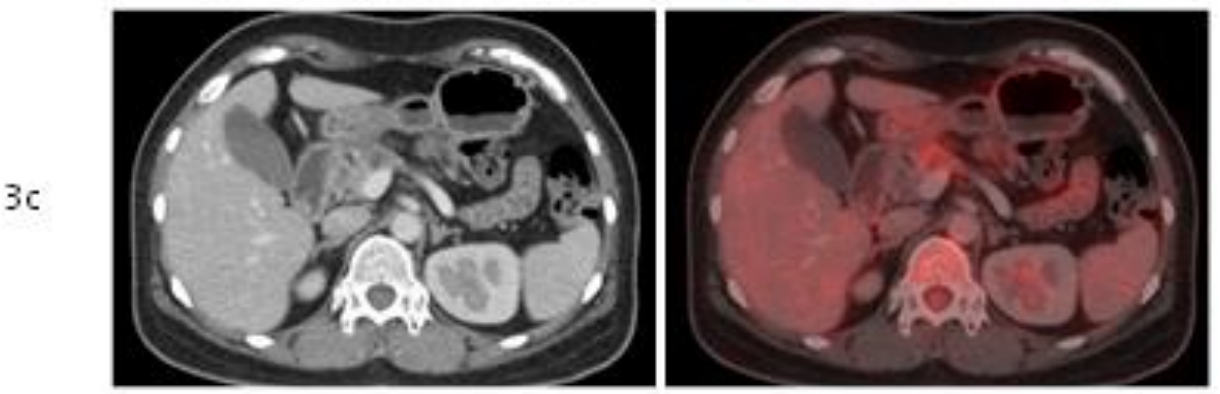

$3 d$
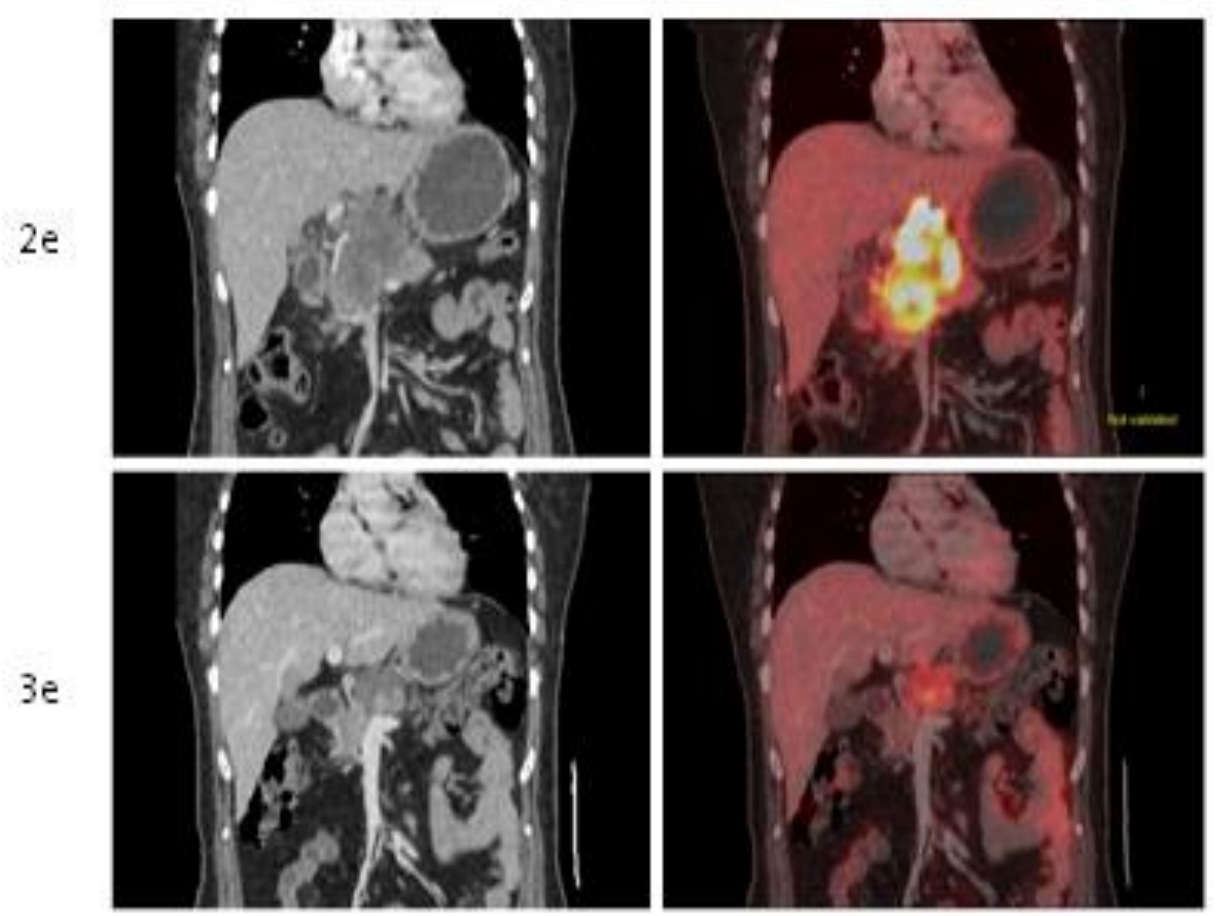

Fig 2a bcd \& \& f: CT \& fused PET CT ima ges, axa l and coronal staging scan. Fig a \& b show hetelogenes ut/ enha ixing maz kesion inol ing the pancreatic head, nech a nd proximal body, with centra l necrosie alow ing increased FDG upta le. Infiltation of first part of duodenum seen (bhch a now). Fig $2 \mathrm{E} \& \mathrm{~d}$ show the tumor infiltating the pybric antı un (white anow). Fig $2 \& \&$ fcoronal ima ges showing infiltation of left b be of liver (astriix).

Fig 3 a bc de \& f: CT \& fused PET CT image, axal and coronal pect chemotherapy scan. Images show

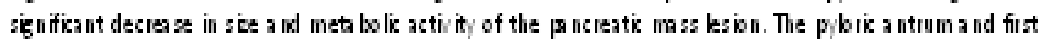
part of duodenum are fies from the kesion. Suggeating a near complete metr bo lic response $t$ trea tme it.

\section{Discussion}

Gastrointestinal lymphomas constitute $10-15 \%$ of all non-Hodgkin lymphomas and $30-40 \%$ of all extranodal lymphomas with Gastrointestinal non-Hodgkin lymphoma (NHL) usually involving the stomach and the small bowel. PPLs are rare. Most cases of pancreatic nonHodgkin lymphoma usually present as a disseminated disease [1]. The most common histologic type of the pancreatic lymphoma is diffuse large B-cell lymphoma [1]. Accounting for $77 \%$ to $80 \%$ of all patients [3]. PPL are also known to present as follicular lymphoma, small lymphocytic 
lymphoma, Burkitt's lymphoma, and rarely as a T cell lymphoma [3]. The clinical presentation of primary pancreatic lymphoma is nonspecific, varying from abdominal pain which is most common presenting symptom , followed by abdominal mass, weight loss, jaundice, acute pancreatitis, small bowel obstruction and diarrhea .The classic symptoms of nodal nonHodgkin's lymphoma, such as fever, chills and night sweats are uncommon [4].

Diagnostic criteria for PPL, as described by Dawson et al. Behrns include (a) mass predominantly located in the pancreas with lymph nodes confined to the peri pancreatic region. (b) neither superficial lymphadenopathy nor enlargement of mediastinal lymph nodes on chest radiography (c) a normal leukocyte count in peripheral blood, and (d) no liver or splenic involvement [4]. These days however CT chest abdomen and/or PET-CT are standard baseline imaging tests, with higher sensitivity to pick up occult or small volume disease in the mediastinum and other sites, as illustrated in our case too.CA 19-9, Lactate dehydrogenase $(\mathrm{LDH})$ and beta-2-microglobulin are essential serum markers for the diagnosis and differential diagnosis of PPL and for differentiating from pancreatic adenocarcinoma [4]. CA 19.9 tends to be raised in adenocarcinoma, while raised LDH would be more in favour of a lymphoma. Imaging studies tend to show a bulky localized tumor in the pancreatic head without significant dilatation of the main pancreatic duct, infrahilar retroperitoneal enlarged lymph nodes and invasive tumor growth not respecting anatomic boundaries with infiltration of surrounding structures. Also the presence of necrosis and calcification is unlikely in NHL [5]. However, the definitive diagnosis of PPL is based on the histopathological and cytopathological examinations [3, 5]. For a definitive diagnosis, CT, or ultrasound-guided fine needle aspiration biopsy is the optimal approach, as it is highly accurate [5]. Pathological evidence is useful not only for the diagnosis, but also for the classification of lymphomas which is mandatory for the choice of chemotherapeutic regimen [6].

Although morphologically different, these two lesions can still diagnosed using IHC which prove to be confirmatory in cases of ambiguity. In patients with aggressive lymphomas FDG-PET is done at baseline for staging, for interim evaluation and to assess response to therapy. It has shown to be a useful prognostic tool to predict relapse risk after the chemotherapy. Poorer clinical outcomes were seen in patients with positive FDG-PET scans as compared to patients with negative scans [6].

Total pancreatectomy (Whipple procedure), which is standard of care for resectable pancreatic adenocarcinomas is considered to have no impact on survival in PPL and, because of associated morbidity, is not generally recommended for diagnosis and treatment of PPL [4,7]. Thus PPL are treated on similar lines to NHL at other sites. The poly-chemotherapy with R- CHOP represents the standard chemotherapy regimen for DLBL treatment with a complete response in $45-53 \%$ of cases and long-term survival of $30-37 \%$ [6]. The role of radiation therapy in PPL remains poorly defined [8]. Bouvet and colleagues have reported use of adjuvant radiation to decrease local failures [9]. Earlier Studies have shown some evidence that an initial surgical resection, when coupled with chemotherapy and radiotherapy, was associated with increased long-term survival of PPL [7], but R-CHOP remains standard for extranodal lymphomas. Prognosis of PPL is significantly better than pancreatic adenocarcinoma, with cure rates of up to $30 \%$ in earlier series compared to less than $5 \% 5$ year survivals for the latter $[10,11,12]$.

\section{Conclusion}

To conclude, PPLs are a class of rare cancers afecting the pancreas, of which DLBL is usually the common variant. However, as efficacious treatments exist for this entity and differ markedly from that of pancreatic adenocarcinom, it is very important that PPLs should be included in the differential when evaluating a probable pancreatic neoplasm.

\section{References}

1. Sugunah Sallapan, Nor Zailin Abu Bakar, Razman Jarmin, Noraidah M Asir, Fazarina Mohammed. (2018) Primary follicular lymphoma of the pancreas: A rare tumour mimicking pancreatic carcinoma. Malaysian J Pathol. 40:359-371.

2. Mukhija D, Nagpal SJS, Sohal DPS. (2019) Epidemiology, Tumor Characteristics, and Survival in Patients with Primary PancreaticLymphoma: A Large Population-based Study Using the SEER Database. Am J Clin Oncol. 42:454-458.

3. Zheng SM, Zhou DJ, Chen YH, Jiang R, Wang YX, Zhang Y et al. (2017) Pancreatic T/histiocyte-rich large B-cell lymphoma: A case report and review of literature. World J Gastroenterol. 23:4467-4472.

4. Saif MW. (2006) Primary pancreatic lymphomas. JOP. 7:262-273.

5. Dinesh M, Shekhar Sharma, Abdul Majeed, Vijaykumar DK, Pavithran K. (2009) Primary pancreatic lymphoma: Report of three cases with review of literature. Indian J Med Paediatr Oncol. $30 ; 20-23$.

6. Constantin A et al. (2018) A Primary Retroperitoneal Diffuse Large B-Cell Lymphoma: A Challenging Diagnosis. Current health sciences journal. 44:392-396.

7. Yu L, Chen Y, Xing L. (2017) Primary pancreatic lymphoma: two case reports and a literature review. Onco Targets Ther. 10:16871694.

8. Shahar KH, Carpenter LS, Jorgensen J, Truong L, Baker K, Teh BS. (2005) Role of radiation therapy in a patient with primary pancreatic lymphoma. Clin Lymphoma Myeloma. 6:143-145.

9. Bouvet M, Staerkel GA, Spitz FR, Curley SA, Charnsangavej C, Hagemeister FB et al. (1998) Primary pancreatic lymphoma. Surgery. 123:382-390.

10. Dawson IM, Cornes JS, Morson BC. (1961) Primary malignant lymphoid tumours of the intestinal tract: Report of 37 cases with a study of factors influencing prognosis. Br J Surg. 4:80-91.

11. Mansour GM, Cucchiaro G, Niotis MT, Fetter BF, Moore J, Rice RR. (1989) Surgical management of pancreatic lymphoma. Arch Surg. 124:1287-1289.

12. Salvatore JR, Cooper B, Shah I, Kummet T. (2000) Primary pancreatic lymphoma: A case report, literature review and proposal for nomenclature. Med Oncol. 17:237-247. 